\title{
SOME NIL RING PROPERTIES RELATED TO $T$-NILPOTENCE
}

\section{B.J. Gardner}

We examine the rings in which every sequence has either a subsequence, or a rearrangement, such that for some $n$ the product of the first $n$ terms in zero.

A ring is left $T$-nilpotent if for every sequence $\left(a_{1}, a_{2}, \cdots\right)$ of its elements there is an index $n$ such that $a_{1} a_{2} \cdots a_{n}=0$. In this note we shall consider two related properties of a ring, which we shall call (s) (for subsequence) and (r) (for rearrangement).

(s) For every sequence $\left(a_{1}, a_{2}, \cdots\right)$ there is a subsequence $\left(a_{\sigma(1)}, a_{\sigma(2)}, \cdots\right)$ and an index $n$ such that $a_{\sigma(1)} a_{\sigma(2)} \cdots a_{\sigma(n)}=0$.

(r) For every sequence $\left(a_{1}, a_{2}, \cdots\right)$ there is a rearrangement $\left(a_{\rho(1)}, a_{\rho(2)}, \cdots\right)$ and an index $n$ such that $a_{\rho(1)} a_{\rho(2)}, \cdots a_{\rho(n)}=0$.

Some years ago Gordon Preston asked the author what sort of rings satisfy (s). We shall show that (s) is equivalent to left $T$-nilpotence; (r) on the other hand, is a bit more difficult to pin down.

It is clear that both (s) and (r) are implied by left $T$-nilpotence and all three properties coincide on commutative rings. By looking at constant sequences we can see that rings with (s) or (r) are nil.

Our demonstration of the equivalence of (s) and $T$-nilpotence relies very much on an attractive theorem (and proof) of Lanski [3]: a semiprime ring cannot be a finite union of right annihilators. This proof in turn makes essential use of Bernhard Neumann's result that if a group is a finite union of cosets it is the union of the ones corresponding to subgroups of finite index [4].

THEOREM 1. A ring satisfies (s) (if and) only if it is left $T$-nilpotent.

Proof: Let $A \neq 0$ have (s). We shall first prove that $A$ is the union of finitely many right annihilators.

Suppose this is not so. We shall denote the right annihilator of an element $a$ by $(a: 0)$. If $0 \neq a_{1} \in A$, then $A \neq\left(a_{1}: 0\right)$ so there exists an $a_{2} \in A$ with $a_{1} a_{2} \neq 0$. Since clearly $a_{2} \neq 0$, we have

$$
A \neq\left(a_{1}: 0\right) \cup\left(a_{2}: 0\right) \cup\left(a_{1} a_{2}: 0\right)
$$

Received 4 December 1991

Copyright Clearance Centre, Inc. Serial-fee code: 0004-9729/92 \$A2.00+0.00. 
Hence for some $a_{3} \neq 0$ we have $a_{1} a_{3}, a_{2} a_{3}, a_{1} a_{2} a_{3} \neq 0$. If now there exist $a_{1}, a_{2}, \cdots, a_{n}$ such that every non-zero "subsequence" of $\left(a_{1}, a_{2}, \cdots, a_{n}\right)$ has nonzero product in the indicated order then $A$ is not the union of the right annihilators of these products, so there exists an $a_{n+1} \in A$ such that $\pi a_{n+1} \neq 0$ for every such product $\pi$. But a product of a "subsequence" of $\left(a_{1}, a_{2}, \cdots, a_{n}, a_{n+1}\right)$ has the form $\pi, \pi a_{n+1}$ or $a_{n+1}$ so all these products are non-zero. But then, inductively, we have a sequence $\left(a_{1}, a_{2}, \cdots\right)$ such that for every subsequence $\left(a_{\sigma(1)}, a_{\sigma(2)}, \cdots\right)$, all products $a_{\sigma(1)} a_{\sigma(2)} \cdots a_{\sigma(n)}$ are non-zero. From this contradiction we infer that $A$ is a union of finitely many right annihilators.

By our remarks above, we can now say that $A$ has elements $b_{1}, b_{2}, \cdots, b_{k} \neq 0$ such that $A=\left(b_{1}: 0\right) \cup\left(b_{2}: 0\right) \cup \cdots \cup\left(b_{k}: 0\right)$ and each $\left(b_{i}: 0\right)$ has finite index in $A^{+}$ (the additive group of $A$ ). We now follow the proof of Lanski's theorem [3]. Let

$$
T=\left(b_{1}: 0\right) \cap\left(b_{2}: 0\right) \cap \cdots \cap\left(b_{k}: 0\right) .
$$

Then $T$ has finite index in $A^{+}$, so $A / T$ is a finite right $A$-module. Let $I$ be the kernel of the ring homomorphism $f: A \rightarrow \operatorname{End}_{\mathrm{z}}(A / T)$ given by

$$
(a+T) f(x)=a x+T
$$

Then $A / I$ is nilpotent as $A$ is nil and $\operatorname{End}_{\mathbf{z}}(A / T)$ is finite. Let $(A / I)^{m}=0$. Then $A^{m} \subseteq I$ so for all $z \in A^{m}$ we have

$$
0=(a+T) f(z)=a z+T
$$

for all $a \in A$, so $A^{m+1} \subseteq T \subseteq\left(b_{1}: 0\right)$. Let $b A^{t+1}=0$ with $b \neq 0$ and $t(\leqslant m)$ as small as possible. Then $b A^{t} \neq 0$ but $b A^{t} A=0$, so $A$ has a non-zero left annihilator.

Thus every non-zero ring with (s) has a non-zero left annihilator. Since clearly (s) is preserved in homomorphic images, it follows (see for example [2], Theorem 1.2) that rings with (s) are left $T$-nilpotent.

It is convenient to consider ( $r$ ) in conjunction with another nil ring property. A ring is $M$-nilpotent if for every double sequence $\cdots, a_{-2}, a_{-1}, a_{0}, a_{1}, a_{2}, \cdots$ of its elements there exist $m, k(k \geqslant 0)$ such that $a_{m} a_{m+1} \cdots a_{m+k}=0$. (See [5].)

PROPOSITION 1. All $M$-nilpotent rings have(r). The converse is false.

Proof: Let $A \neq 0$ be $M$-nilpotent, $\left(a_{1}, a_{2}, \cdots\right)$ a sequence of its elements. From consideration of the double sequence

$$
\left(\cdots, a_{2 n}, a_{2 n-2}, \cdots, a_{6}, a_{4}, a_{2}, a_{1}, a_{3}, \cdots, a_{2 n-1}, \cdots\right)
$$


we see that $a_{2 m} a_{2 m-2} \cdots a_{2} a_{1} a_{3} \cdots a_{2 k-1}=0$ for some $m, k$. There is clearly no loss of generality in assuming $m=k$. But then the product of the first $4 m-1$ terms of

$$
\left(a_{2 m}, a_{2 m-2}, \cdots, a_{2}, a_{1}, a_{3}, \cdots, a_{2 m-1}, a_{2 m+1}, a_{2 m+2}, \cdots\right)
$$

is 0 . Hence $A$ has (r).

We now show that if a ring $A$ has an ideal $I$ such that $A / I$ and $I$ both satisfy (r), then $A$ does also. It has been shown by Sands [5, Example 2] that the class of $M$-nilpotent rings does not have this property, so it follows that (r) does not imply $M$-nilpotence.

Let $\left(a_{1}, a_{2}, \cdots\right)$ be a sequence of elements of $A$. Let $\bar{x}=x+I$ for $x \in A$. Then as $A / I$ satisfies (r) there are distinct indices $i_{1}, i_{2}, \cdots, i_{n_{1}}$ such that $\bar{a}_{i_{1}} \bar{a}_{i_{2}} \cdots \bar{a}_{i_{n_{1}}}=0$, that is, $a_{i_{1}} a_{i_{2}} \cdots a_{i_{n_{1}}} \in I$. Now consider $\left(a_{i_{n_{1}}+1}, a_{i_{n_{1}}+2}, \cdots\right)$. In the same way, there exist distinct indices $j_{1}, j_{2}, \cdots, j_{n_{2}}$ such that $a_{i_{n_{1}}+j_{1}} a_{i_{n_{1}}+j_{2}} \cdots a_{i_{n_{1}}+j_{n_{2}}} \in I$. Let $b_{1}=a_{i_{1}} a_{i_{2}} \cdots a_{i_{n_{1}}}, b_{2}=a_{i_{n_{1}}+j_{1}} a_{i_{n_{1}}+j_{2}} \cdots a_{i_{n_{1}}}+j_{n_{2}}$. Proceeding in this way, we obtain a sequence $\left(b_{1}, b_{2} \cdots\right)$ of elements of $I$, each term of which is a product of a set of distinct terms of $\left(a_{1}, a_{2}, \cdots\right)$ and such that these sets of terms are pairwise disjoint. (More precisely it is the sets of indices that are pairwise disjoint.) But $I$ has (r) so there are indices $k_{1}, k_{2}, \cdots, k_{n}$ such that $b_{k_{1}} b_{k_{2}} \cdots b_{k_{n}}=0$. But this product is clearly the product of finitely many terms of $\left(a_{1}, a_{2}, \cdots\right)$ and hence of the first few terms of a rearrangement of this sequence. Hence $A$ has (r).

Sands [5] has introduced the middle annihilator of a ring $A$ : the set $\{a \in A: A a A=$ 0 ) and characterised $M$-nilpotent rings as those for which all non-zero homomorphic images have non-zero middle annihilators. This is analogous to the characterisation of left $T$-nilpotence alluded to above, using left annihilators. It would be interesting to have a similar description of the rings with ( $r$ ). We do not have such a description but can get a related upper bound on the class of rings with (r).

For an ordered pair $(a, b)$ of elements of a ring $A$ we define the middle annihilator $\alpha(a, b)$ to be $\{r \in A: a r b=0\}$.

Proposition 2. If a ring $A$ satisfies (r) then every non-zero homomorphic image of $A$ is a finite union of middle annihilators of ordered pairs of non-zero elements.

Proof: Let $A \neq 0$ be a ring which is not a finite union of middle annihilators. We first note that as $\alpha(a, b)$ contains both $(a: 0)$ and $(0: b)$ (left annihilator), $A$ is not a finite union of left, right and middle annihilators of non-zero elements or ordered pairs of such. If $0 \neq a_{1} \in A$, then as noted above, $A \neq\left(0: a_{1}\right) \cup\left(a_{1}: 0\right)$, so there exists $a_{2} \in A$ such that $a_{2} a_{1} \neq 0$ and $a_{1} a_{2} \neq 0$. Thus no rearrangement of $\left(a_{1}, a_{2}\right)$ has zero product. If now there exist $a_{1}, a_{2}, \cdots, a_{n} \in A$ (necessarily in $A \backslash\{0\}$ ) such that no product $\pi$ of elements $a_{i}$ with distinct values of $i$ is 0 , then

$A \neq \cup\left\{\alpha\left(\pi_{1}, \pi_{2}\right): \pi_{1}, \pi_{2}\right.$ are products of elements $a_{i}$ with distinct values of $\left.i\right\}$. 
Hence there exists $a_{n+1} \in A$ such that $\pi a_{n+1} \pi^{\prime} \neq 0$ for all $\pi, \pi^{\prime}$, and hence $\pi a_{n+1} \neq 0$ and $a_{n+1} \pi^{\prime} \neq 0$ for all $\pi, \pi^{\prime}$. But every product of factors from $\left\{a_{1}, a_{2}, \cdots, a_{n}, a_{n+1}\right\}$ with different indices must have the form $\pi a_{n+1} \pi^{\prime}$ or $\pi a_{n+1}$ or $a_{n+1} \pi^{\prime}$ or $\pi$. Thus inductively we have a sequence $\left(a_{1}, a_{2}, \cdots\right)$ such that $a_{i_{1}} a_{i_{2}} \cdots a_{i_{n}} \neq 0$ for all distinct $i_{1}, i_{2}, \cdots, i_{n}$. This means that $A$ does not satisfy (r).

Thus every ring with (r) is a finite union of middle annihilators and since $(r)$ is preserved under homomorphic images, the result follows.

The converse to Proposition 2 is false, even with nil as an additional requirement.

EXAMPLE: Let $R$ be a nil ring with central element $c$ such that $c^{n+1}=0 \neq$ $c^{n}$. Then $R=\alpha\left(c^{n}, c\right)$. Thus a nil ring such that every non-zero homomorphic image has non-zero centre (for example a commutative nil ring) satisfies the conclusion of Proposition 2, while as we have noted already, for commutative rings, (r) and $T$ nilpotence are equivalent.

Note also that even a prime simple ring can be a finite union of middle annihilators.

EXAMPLE: Let $M_{2}\left(\mathbb{Z}_{2}\right)$ denote the ring of $2 \times 2$ matrixes over $\mathbb{Z}_{2}$. Then

$$
\begin{array}{r}
M_{2}\left(\mathbb{Z}_{2}\right)=\alpha\left(\left[\begin{array}{ll}
0 & 1 \\
0 & 0
\end{array}\right],\left[\begin{array}{ll}
0 & 1 \\
0 & 0
\end{array}\right]\right) \cup \alpha\left(\left[\begin{array}{ll}
0 & 1 \\
0 & 0
\end{array}\right],\left[\begin{array}{ll}
0 & 0 \\
1 & 0
\end{array}\right]\right) \cup \alpha\left(\left[\begin{array}{ll}
0 & 0 \\
1 & 0
\end{array}\right],\left[\begin{array}{ll}
0 & 1 \\
0 & 0
\end{array}\right]\right) \\
\cup \alpha\left(\left[\begin{array}{ll}
0 & 0 \\
1 & 0
\end{array}\right],\left[\begin{array}{ll}
0 & 0 \\
1 & 0
\end{array}\right]\right) \cup \alpha\left(\left[\begin{array}{ll}
1 & 0 \\
1 & 0
\end{array}\right],\left[\begin{array}{ll}
1 & 0 \\
1 & 0
\end{array}\right]\right) .
\end{array}
$$

It is natural to ask whether or not rings with ( $r$ ) must be prime radical. Since ( $r$ ) is preserved in homomorphic images, the question has an affirmative answer if and only if there are no prime rings with (r). While this seems highly likely, we have been unable to prove it. We do have some information, however.

PROPOSITION 3. If a prime ring has ( $\mathrm{r}$ ) then it has prime characteristic and is not an algebra over an infinite field.

Proof: Suppose $A$ is a prime ring containing non-zero elements $a_{1}, b_{1}, a_{2}, b_{2}$, $\cdots, a_{n}, b_{n}$ such that

$$
A=\alpha\left(a_{1}, b_{1}\right) \cup \alpha\left(a_{2}, b_{2}\right) \cup \cdots \cup \alpha\left(a_{n}, b_{n}\right)
$$

Then by the result from [4] again, we can assume each $\alpha\left(a_{i}, b_{i}\right)$ (not necessarily a one-sided ideal, but certainly a subgroup) has finite index in $A^{+}$. Now $A$ is a prime ring, so either $A^{+}$is torsion-free or $p A=0$ for some prime $p$. In the former case, let $\alpha\left(a_{1}, b_{1}\right)$ have index $m$ in $A^{+}$. Then for all $r \in A$ we have $m r \in \alpha\left(a_{1}, b_{1}\right)$, that is, $a_{1} m r b_{1}=0$. But then $a_{1} A\left(m b_{1}\right)=0$ so as $A$ is prime, $a_{1}=0$ or $m b_{1}=0$ and thus, as $A^{+}$is torsion-free, $a_{1}=0$ or $b_{1}=0$, a contradiction. If $p a=0$, then $A$ is a 
$\mathbb{Z}_{p}$-algebra. Let $K$ be a field of characteristic $p$ such that $A$ is a $K$-algebra. Then if $t \in \alpha\left(a_{1}, b_{1}\right)$ and $u \in K$, we have

$$
a_{1}(u t) b_{1}=a_{1} u\left(t b_{1}\right)=u\left(a_{1} t b_{1}\right)=0,
$$

so $\alpha\left(a_{1}, b_{1}\right)$ is a $K$-subspace of $A$. Suppose $K$ is infinite. Then the index of $\alpha\left(a_{1}, b_{1}\right)$ in $A^{+}$is the cardinality of a complementary subspace, so this must be 1 , that is, $A=\alpha\left(a_{1}, b_{1}\right)$, that is, $a_{1} A b_{1}=0$. But $A$ is prime so $a_{1}$ or $b_{1}=0$ and we again have a contradiction.

COROLlary. If a ring has torsion-free additive group and satisfies $(\mathrm{r})$, it is a prime radical ring.

Proof: Let $R$ satisfy (r) and have torsion-free additive group. Let $D(R)$ denote the divisible hull of $R$. This is a ring in the obvious way and is a $\mathbb{Q}$-algebra (see [1], p.284). Let $\left(a_{1}, a_{2}, \cdots\right)$ be a sequence in $D(R)$. Then for each $i$ there is a non-zero integer $k_{i}$ such that $k_{i} a_{i} \in R$. Since $R$ satisfies $(\mathrm{r})$, the sequence $\left(k_{1} a_{1}, k_{2} a_{2}, \cdots\right)$ has a rearrangement $\left(k_{\rho(1)} a_{\rho(1)}, k_{\rho(2)} a_{\rho(2)}, \cdots\right)$ such that

$$
k_{\rho(1)} k_{\rho(2)} \cdots k_{\rho(n)} a_{\rho(1)} a_{\rho(2)} \cdots a_{\rho(n)}=k_{\rho(1)} a_{\rho(1)} k_{\rho(2)} a_{\rho(2)} \cdots k_{\rho(n)} a_{\rho(n)}=0
$$

for some $n$. Since $D(R)$ is torsion-free, it follows that $a_{\rho(1)} a_{\rho(2)} \cdots a_{\rho(n)}=0$. Thus $D(R)$ satisfies $(\mathrm{r})$. Since $D(R)$ is divisible, any prime homomorphic image that it might have would need to be torsion-free and satisfy $(r)$. But by Proposition 3 such prime rings do not exist. Hence $D(R)$ is a prime radical ring, so $R$ is too.

\section{REFERENCES}

[1] L. Fuchs, Infinite abelian groups Vol.II (Academic Press, New York and London, 1973).

[2] B.J. Gardner, 'Some aspects of T-nilpotence', Pacific J. Math. 53 (1974), 117-130.

[3] C. Lanski, 'Can a semi-prime ring be a finite union of right annihilators?', Canad. Math. Bull. 33 (1990), 126-128.

[4] B.H. Neumann, 'Groups covered by permutable subgroups', J. London Math. Soc. 29 (1954), 236-248.

[5] A.D. Sands, 'On $M$-nilpotent rings', Proc. Roy. Soc. Edinburgh 93A (1982), 63-70.

\footnotetext{
Department of Mathematics

University of Tasmania

GPO Box 252C

Hobart, Tas. 7001

Australia
} 